\title{
Quantification of the chemical composition variations of tumors in photothermal therapy by photoacoustic spectroscopy: An in vitro study
}

\author{
Yubin Liu ${ }^{\mathrm{a}, \mathrm{b}}$, Zhifang $\mathrm{Li}^{\mathrm{b}}$, Hui $\mathrm{Li}^{\mathrm{b},{ }^{*}}$ and Zhen Yuan ${ }^{\mathrm{a},{ }^{*}}$ \\ ${ }^{a}$ Bioimaging Core, Faculty of Health Sciences, University of Macau, Macau SAR, China \\ ${ }^{\mathrm{b}}$ College of Photonic and Electronic Engineering, Fujian Normal University, Fuzhou 350007, China
}

\begin{abstract}
In this study, the change of tumors' chemical composition in the temperature range of $20 \sim 70^{\circ} \mathrm{C}$ is quantified for photothermal tumor therapy by photoacoustic spectroscopy (PAS) with the wavelengths of $700 \sim 1000 \mathrm{~nm}$. Based on the measured photoacoustic signals, two absorption peaks at the wavelengths of $750 \mathrm{~nm}$ and $950 \mathrm{~nm}$ are identified. It is also observed that high temperature $\left(>55^{\circ} \mathrm{C}\right)$ is able to induce the physical and chemical degeneration of tumors. According to the in vitro tests, a new chemical species, met-hemoglobin, which is absent in normal blood, is generated at high temperature with enhanced near-infrared absorption.
\end{abstract}

Keywords: PAS, photothermal therapy, photoacoustic signals, hemoglobin

\section{Introduction}

Photoacoustic spectroscopy (PAS) is a spectral approach that allows for direct measurements of tissue chromophores and other physiology parameters using multiple-wavelength laser illumination. PAS is based upon the photoacoustic effect, in which the laser-produced temperature rise and subsequent thermoelastic expansion of tissues generate acoustic waves which is detected by ultrasound transducers along multiple boundary positions. PAS was first used to measure the physical parameters of gas and now has become an essential tool to quantify tissue physiological functions, disease progression, responses to intervention and photothermal cancer therapy [1-6]. If we search the term "photoacoustic spectroscopy" using PubMed, we will find that the number of publications on PAS has increased from several in 1970 to almost a thousand to date.

In addition, photothermal therapy is a laser-based mini-invasive technique with extensive clinical and pre-clinical applications. It utilizes high continuous light intensity to irradiate the diseased tissues,

\footnotetext{
${ }^{*}$ Corresponding author: Hui Li, College of Photonic and Electronic Engineering, Fujian Normal University, Fuzhou 350007, China. Tel.: 059122868133; Fax: 059122868133; E-mail: hli@fjnu.edu.cn.

Zhen Yuan, Bioimaging Core, Faculty of Health Sciences, University of Macau, Macau SAR, China. Tel.: 85388224989; Fax: 85388222314; E-mail: zhenyuan@umac.mo.
} 
which is able to convert light energy to heat, causing localized thermal destruction of tissues $[7,8]$. The definition of the critical temperature of hyperthermia, coagulation, vaporization, carbonization and fusion induced by laser has been proposed for in vivo study of photothermal cancer therapy [9]. Based on this, the temperature ranging from $42^{\circ} \mathrm{C}$ to $50^{\circ} \mathrm{C}$ is generally called hyperthermia, in which the tissues or organs will become necrotic if the laser irradiation lasts for a few minutes. And if temperature is over $60^{\circ} \mathrm{C}$, the irradiation will lead to condensation of tissues and necrocytosis of cells as well as the denaturation of protein and collagen. This study will utilize PAS to measure and monitor the physiology parameters and chemical composition variations of tumors during photothermal therapy. Due to the use of multiple laser wavelengths, PAS is able to provide more accurate measurements of functional parameters including changes of hemoglobin and other species. Finally, in vitro tests will be used to demonstrate and validate PAS method for photothermal therapy.

\section{Materials and methods}

\subsection{The theoretical foundation of $P A S$}

Based on the photoacoustic effect, PAS uses a short pulse laser to illuminate the absorbers in tissues to generate the acoustic waves. The measured photoacoustic (PA) pressure that satisfies the temporal stress confinement is written as [1-5]:

$$
P(z)=\left(\beta c^{2} / C_{p}\right) \mu_{a} F(z)=\left(\beta c^{2} / C_{p}\right) \mu_{a} F_{0} \exp \left(-\mu_{a} z\right)
$$

in which $\beta$ is the expansion coefficient, $c$ is the speed of sound, $C_{p}$ is the specific heat, $\mu_{a}$ is the absorption coefficient of medium, $F_{0}$ is the initial optical fluence, and $z$ is the one-dimensional incident depth at position $z$ along the laser illumination direction. If the optical fluence $F$ at different penetration depth is defined, the following equation will be obtained:

$$
F=F_{0} \exp \left(-\mu_{a} z\right)
$$

Additionally, the light absorbed energy density is written as:

$$
G=\mu_{a} F
$$

It is noted from Eqs. (1)-(3) that the PA signal (pressure) is proportional to the light absorbed energy density $G$ along the laser source incident direction: the maximum light energy is converted into thermal energy, i.e., the strongest PA signal. Here introduces a Grüneisen parameter $\Gamma$,

$$
\Gamma=\beta c^{2} / C_{p}
$$




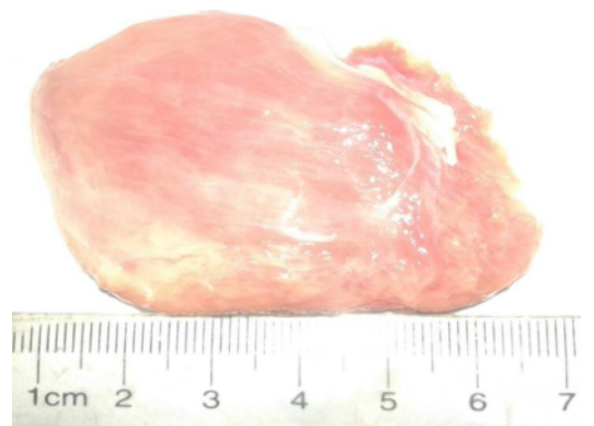

(a)

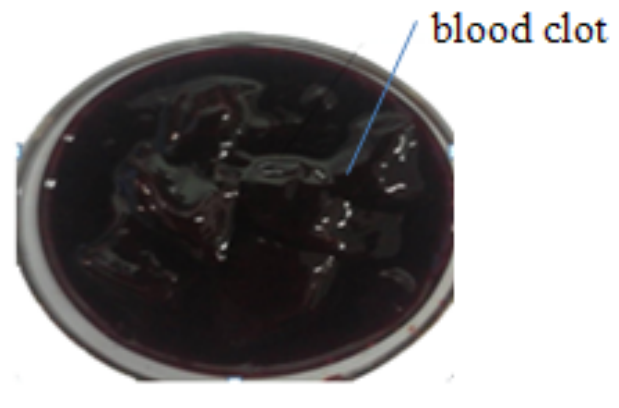

(b)

Fig. 1. (a) The chicken breast and (b) the blood clot.

For soft tissues with temperature $T$ between $10-55^{\circ} \mathrm{C}$, the expansion coefficient $\beta$ is linearly proportional to $T[10,11]$,

$$
\Gamma=A+B T
$$

in which $A$ and $B$ are constants. As such, Eq. (1) can be further written as:

$$
P(z)=\Gamma \mu_{a} F(z)=(A+B T(z)) \mu_{a} F(z)=(A+B T(z)) \mu_{a} F_{0} \exp \left(-\mu_{a} z\right)
$$

in which $T(\mathrm{z})$ is the temperature distribution of tissues. If the initial temperature distribution of tissues $T_{0}$ is assumed, Eq. (7) can be achieved:

$$
P(z)_{T=T_{0}}=\left(A+B T_{0}\right) F_{0} \exp \left(-\mu_{a} z\right)
$$

In terms of Eqs. (6) and (7), the following Eq. (8) is obtained,

$$
T(z)=\left(A+B T_{0}\right) P(z) /\left(B P(z)_{T=T_{0}}\right)-A / B=C+D P(z) / P(z)_{T=T_{0}}
$$

in which $C=A / B, D=\left(A+B T_{0}\right) / B$ are parameters that are dependent on tissue properties, and $P(z)_{T=T_{0}}$ is the PA pressure profile recorded at the initial temperature. It is noted from Eq. (8) that the PA signal amplitudes is linearly proportion to the temperature. However, it only works well when the temperature is between $10-55^{\circ} \mathrm{C}$. When the temperature is above $55^{\circ} \mathrm{C}$, photochemical and photothermal modifications will exert an impact on the chemical constituents of blood, which will be validated by our new findings in Section 3. By querying data, we have found that a new chemical 


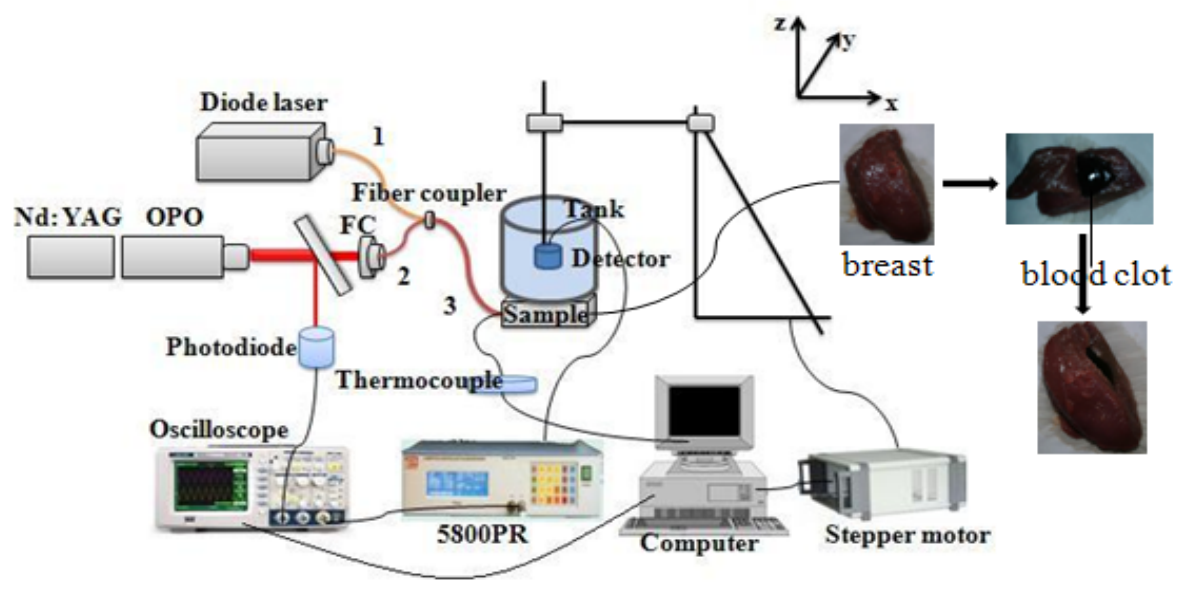

(a)

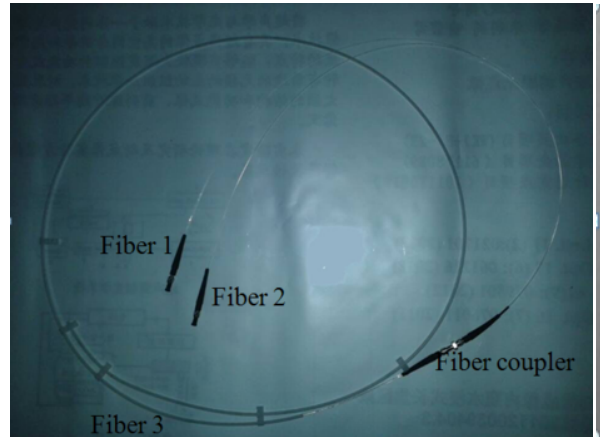

(b)

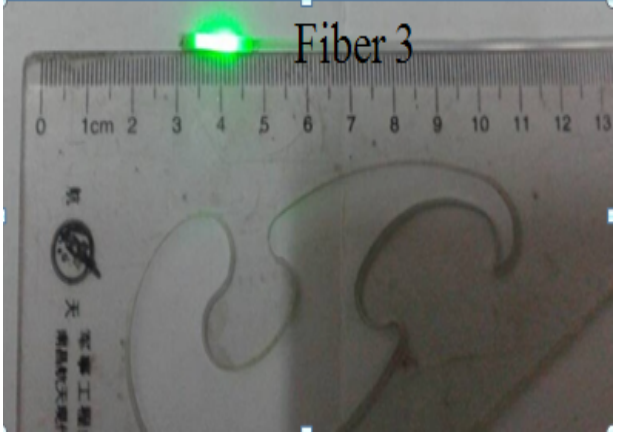

(c)

Fig. 2 (a) The experimental setup for exploring the physical and chemical mechanism of photothermal tumor therapy by PAS. (b) The diagram of two fiber coupling into a fiber by SMA interface. (c) The diagram of optical fiber dispersion.

species called met-hemoglobin is generated, which is closely related with the enhanced near-infrared absorption [12].

\subsection{Materials}

For the in vitro test, the blood clot as tumor simulation samples was buried inside the chicken breast, as plotted in Figure 1.

The experimental setup for PAS of photothermal tumor therapy is shown in Figure 2. The Nd: YAG laser (Surelite I-10, Continuum) with Optical Parametric Oscillator (OPO) (Surelite OPO plus, Continuum) was used for optoacoustic wave generation with a wavelength of $700 \sim 2000 \mathrm{~nm}$, a repetition frequency rate of $10 \mathrm{~Hz}$, a pulse width of $5 \mathrm{~ns}$ and the output energy of about $4 \mathrm{~mJ}$. The diode laser treatment apparatus (ML-4030D) were used for photothermal tumor therapy, which could provide the power range of $1 \sim 30 \mathrm{~W}$ with the adjustable period of $0.5 \mathrm{~W}$ and the output wavelength of $810 \mathrm{~nm}$. The fiber 1 was connected to the diode laser with the core diameter of $400 \mu \mathrm{m}$ and maximum power threshold of $30 \mathrm{~W}$. The fiber 2 was linked to the OPO laser by the FC interface, and the core 
diameter of fiber 2 is $600 \mu \mathrm{m}$ and the maximum power threshold is $6 \mathrm{~W}$. The light beam from OPO is divided into two beams by splitter mirror: one is received by photodiode, displayed on the oscilloscope and then stored by a computer for calibration; the other one just focuses on the port of fiber 2 through a short focal length lens. Fiber 1 and fiber 2 were coupled by SMA interface formation of fiber 3, which had both the therapy and monitor functions, as displayed in Figures 2(b) and 2(c). The transmission wavelengths of optical fibers are between 500 and $2000 \mathrm{~nm}$. The dispersion end (length of $1.5 \mathrm{~cm}$ ) of fiber 3 and the thermode (core diameter of $0.3 \mathrm{~mm}$ ) were inserted into the tumor location of the sample. The upper surface of the sample was coupled with the soffit of the water bank by plastic wrap to reduce the sound attenuation between different media. The depth-resolved photoacoustic signals from the sample were collected by focused water immersion ultrasound transducer (Panametrics-NDT): the bandwidth of transducer ranges from 3.7 to $6.14 \mathrm{MHz}$, the center frequency is $5 \mathrm{MHz}$ and the water path is $25.654 \mathrm{~mm}$, which means that the relative position of the probe with respect to the sample is about $25 \mathrm{~mm}$. Then the signals were transferred to the ultrasonic receiver (5800 R, Parametric-NDT) for amplitude limit, filtering and amplifying. Finally, the signals were showed on the digital oscilloscope (TDS3054C, Tektronix). In order to improve the signal-to-noise ratio, the signals on the digital oscilloscope were taken averages of 16 times and then saved in the computer for the follow-up data processing. The stepper motor (SC300-2B, Zolix) could accurately control bracket by driving the electronic translation machine (TSA200-B, Zolix), for the 2D scanning of the sample.

\section{Results}

Figure 3 plots the near-infrared absorption spectra of the tumor simulation sample at various temperatures, in which $\mathrm{x}$-axis represents different wavelengths while $\mathrm{y}$-axis represents the photoacoustic signal amplitude with unit $\mathrm{mV}$ at different temperatures. It is observed from Figure 3 that there are two absorption peaks of blood: one is at $750 \mathrm{~nm}$ and the other is at $950 \mathrm{~nm}$. This observation is in good agreement with the previous findings [13-15].

Figure 4 presents the relationship between the photoacoustic signals and temperatures at the wavelengths of $750 \mathrm{~nm}$ (Figure 4(a)) and $950 \mathrm{~nm}$ (Figure 4(b)) for two absorption peaks of blood in Figure 3. It is observed from Figure 4 that when the temperature is in the range of $20 \sim 55^{\circ} \mathrm{C}$, a linear relationship exists between the photoacoustic signal amplitudes and the temperatures. However, this is not the case for the temperature over $55^{\circ} \mathrm{C}$, where signals are increasing greater and quicker compared with those from $20 \sim 55^{\circ} \mathrm{C}$. In addition, according to the previous findings [10], when temperature is over $60^{\circ} \mathrm{C}$, the irradiation will lead to condensation of tissues and necrocytosis of cells as well as the denaturation of protein and collagen. These results and findings suggested that a new chemical species was produced and the linear relation between the signals and the temperature was no longer kept. By querying data, the new chemical species should be met-hemoglobin because the met- hemoglobin is implicated in the enhanced near-infrared absorption of blood [12-15]. And we believe the methemoglobin has larger absorption coefficient when the wavelength of pulsed laser source is larger than $600 \mathrm{~nm}$ [16] In particular, several studies have demonstrated that in photothermal therapy the laser energy can alter the absorption characteristics of blood [17], in which the conversion of blood to a combination of met-hemoglobin and thrombus temporarily increases absorbance by approximately 3-5 times over that of normal blood $[18,19]$. Therefore, the mechanism and findings from previous work are in good agreement with ours. 


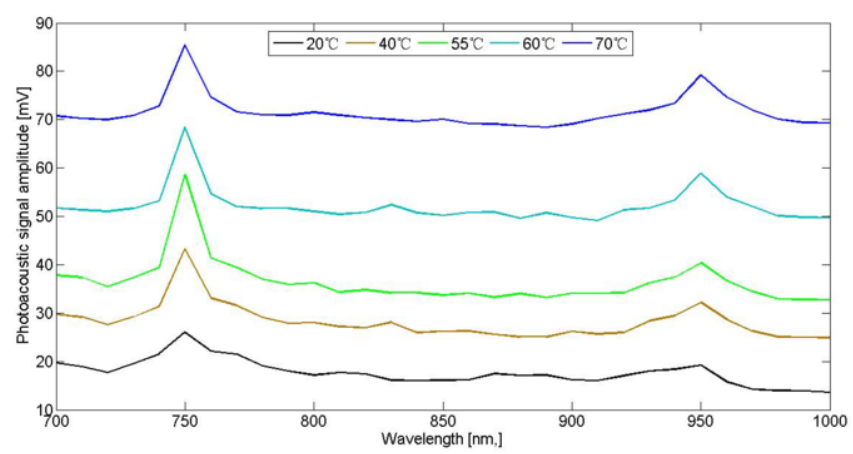

Fig. 3. The near-infrared absorption spectra of tumor simulation sample at various temperatures.

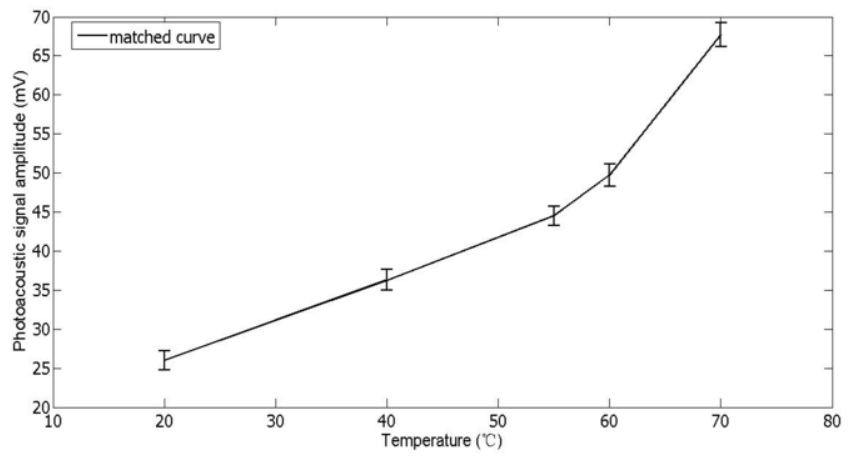

(a)

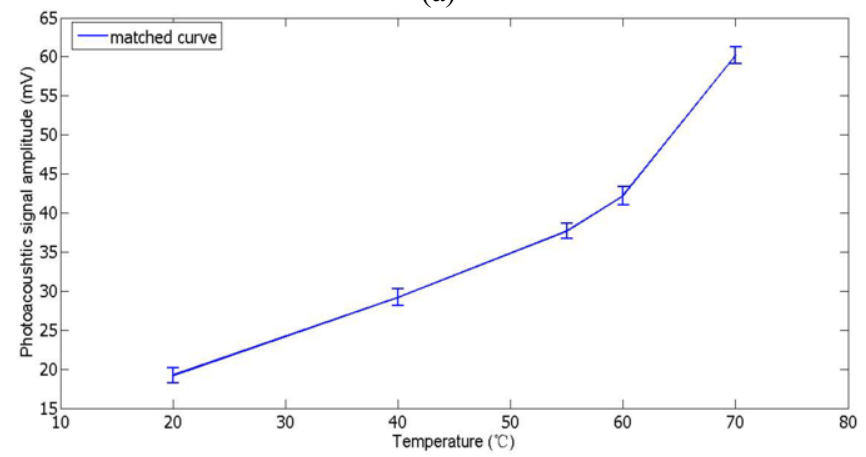

(b)

Fig. 4. (a) The relationship between photoacoustic signal amplitudes and temperatures at wavelengths $750 \mathrm{~nm}$; (b) $950 \mathrm{~nm}$.

Figures 5(a) and 5(b) displays the 2D scanning image of blood clot at wavelengths $750 \mathrm{~nm}$ and 950 $\mathrm{nm}$, respectively, with temperature at $60^{\circ} \mathrm{C}$, in which the x-axis is the sweep point (every bit of the step length is $0.1 \mathrm{~mm}$ and the total scanning length is $3 \mathrm{~cm}$ ) while the y-axis represents the sampling numbers on the oscilloscope (every 1000 points are $2 \mu \mathrm{s}$ ). From Figure 5, the signals are in the range of point 50 to point 200, and the length of dispersion end of fiber 3 is $1.5 \mathrm{~cm}$, which demonstrates that 

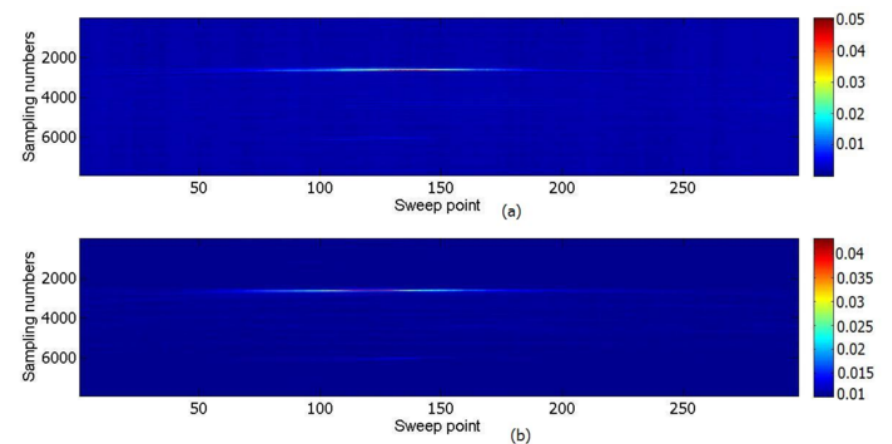

Fig. 5. (a) The 2D scanning image of blood clot with $750 \mathrm{~nm}$ at $60^{\circ} \mathrm{C}$; (b) with $950 \mathrm{~nm}$ at $60^{\circ} \mathrm{C}$.

the signals are from the excitation light source. Moreover, the strongest signals were generated by the blood clot. The grey values of image represent the information of photoacoustic signals and the values of color bar are equivalent to the amplitude of the photoacoustic signal. One can see from Figure 5 that the maximum amplitude of photoacoustic signal is $0.0497 \mathrm{~V}$ with the wavelength of $750 \mathrm{~nm}$ at $60^{\circ} \mathrm{C}$, which also correlates well with the findings in Figure 3. If the relationship between the amplitude of photoacoustic signal and temperature is quantified, the grey-scale map of photoacoustic signal can be transformed into the image of temperature distribution.

\section{Discussion}

This study explored the photochemical degeneration of tumors during photothermal therapy by PAS. Two absorption peaks of blood were observed in vitro: one is $750 \mathrm{~nm}$ and the other is $950 \mathrm{~nm}$. When the temperature is below $55^{\circ} \mathrm{C}$, the two peaks occur due to the absorption of deoxy-hemoglobin, in which the photoacoustic signal amplitudes are proportional to the temperatures. However, when the temperature was over $55^{\circ} \mathrm{C}$, a new chemical species was generated with the enhanced near-infrared absorption, destroying the linear relationship between the signals and the temperatures. In addition, 2D scanning images of the blood clot were also obtained at the wavelengths of $750 \mathrm{~nm}$ and $950 \mathrm{~nm}$ with temperature of $60^{\circ} \mathrm{C}$.

A series of in vitro tests are conducted in this study to evaluate the performance of PAS systems. Though it works very well in terms of the quantitative findings, the controlled environments of in vitro tests are different from that of the non-controlled in vivo cases. As such, further investigations are required to extend the present study to in vivo pre-clinical small animal studies for cancer early detection and treatment. In addition, functional near infrared spectroscopy (fNIRS) can also quantify the physiological parameters of biological tissues [20-22]. However due to the low resolution of the diffuse light, the quantitative accuracy of PAS is much better than that from fNIRS, particularly for photothermal therapy related studies.

Additionally, our further investigation will be focused on quantifying the chromophore concentrations including oxy-hemoglobin concentration and deoxy-hemoglobin concentrations, and now efforts are spared to need incorporate the photon diffuse equation to separate the optical absorption coefficient from photon fluence [3]. 


\section{Acknowledgment}

This project is sponsored in part by Fujian provincial key program of science and technology (No. 2011Y0019), National Natural Science Foundation of China (No. 61178089/81201124). This research was also supported by SRG2013-00035-FHS Grant and MYRG2014-00093-FHS Grant from University of Macau in Macau.

\section{References}

[1] A. Rosencwaig and J. Gersho, Theory of the photoacoustic effect with solids, J. Appl. Phys. 47 (1976), 64-69.

[2] W.R. Harshbarger and M.B. Robin, The optoacoustic effect: revival of an old technique for molecular spectroscopy, Acc. Chem. Res. 6 (1973), 329-334.

[3] Z. Yuan, X. Li and L. Xi, Listening to light scattering in turbid media: quantitative optical scattering imaging using photoacoustic measurements with one-wavelength illumination, J. Opt. 16 (2014), 065301-065309.

[4] Christoph Haisch, Photoacoustic spectroscopy for analytical measurements, Meas. Sci. Technol. 23 (2012), 012001012008.

[5] P. Poulet, J. Chambron and R. Unterreiner, Quantitative photoacoustic spectroscopy applied to thermally thick samples, J. Appl. Phys. 51 (1980), 1738-1741.

[6] Deepak V. Bageshwar, Avinash S. Pawar, Vineeta V. Khanvilkar and Vilasrao J. Kadam, Photoacoustic spectroscopy and its applications-A tutorial review, Eurasian J. Anal. Chem. 5 (2010), 187-203.

[7] Jignesh Shah, Suhyun Park and Salavat Aglyamov et al., Photoacoustic imaging and temperature measurement for photothermal cancer therapy, Journal of Biomedical Optics 13 (2008), 034024.

[8] K. Ivarsson, L. Myllymaki, K. Jabsner et al., Heat shock protein 70 (HSP70) after laser thermotherapy of an adenocarcinoma transplanted into rat liver, Anticancer Res. 23 ( 2003), 3703-3712.

[9] Markolf H. Niemaz et al., Laser-Tissue Interactions: Fundamentals and Applications, Springer Science \& Business Media, 2004.

[10] J. Shah, S. Park and S. Aglyamov et al., Photoacoustic imaging and temperature measurement for photothermal cancer therapy, J. Biomed. Opt. 13 (2008), 034024-034032.

[11] I.V. Larina, L.V. Larin and R.O. Esenaliev, Real-time optoacoustic monitoring of temperature in tissues, J. Phys. D: Appl. Phys. 38 (2005), 2633-2639.

[12] Jennifer Kehlet Barton, George Frangineas, Herbert Pummer and John F. Black, Cooperative phenomena in two-pulse, two-color laser photocoagulation of cutaneous blood vessel, Photochemistry and Photobiology 73 (2001), 642-650.

[13] Lorenzo Cordone, Antonio Cupane, Maurizio Leone and Eugenio Vitrano, Optical absorption spectra of deoxyhemoglobin and oxyhemoglobins in the temperature range 300-20 K relation with protein dynamics, Biophysical Chemistry 24 (1986), 259-275.

[14] William A. Eaton and James Hofrichter, Polarized absorption and linear dichroism spectroscopy of hemoglobin, Methods Enzymol. 76 (1981), 175-261.

[15] William A. Eaton, Louise Karle Hanson, P.J. Stephens, J.C. Sutherland and J.B.R. Dunn, Optical spectra of oxy- and deoxyhemoglobin, J. Am. Chem. Sot. 100 (1978), 4991-5003.

[16] O. Siggaard-Andersen, B. Norgaard-Pedersen and J. Rem, Hemoglobin pigments spectrophotometric determination of oxy-, carboxy-, met-, and sulfhemoglobin in capillary blood, Clinica. Chimica. Acta. 72 (1972), 85-100.

[17] J.F. Black and J.K. Barton, Time-Domain optical and thermal analysis of blood undergoing laser photocoagulation, Proc. of Laser-Tissue Interaction XII: Photochemical, Photothermal, and Photomechanical 4257 (2001), 341-354.

[18] J.T. Kuenstner and K.H. Norris, Spectrophotometry of human hemoglobin in the near infrared region from 1,000 $\mathrm{nm}$ to 2,500 nm, Journal of Near Infrared Spectroscopy 2 (1994), 59-65.

[19] M. Heger et al., Towards optimization of selective photothermolysis: prothromic phermaceutical agents as potential adjuvants in laser treatment of port wine stains, Thromb. Haemost. 93 (2005), 242-256.

[20] Z. Yuan, A spatiotemporal and time frequency analysis of fNIRS brain signals using ICA method, Journal of Biomedical Optics 18 (2013), 106011-106018.

[21] Z. Yuan, Combing ICA and Granger causality to capture brain network dynamics with fNIRS measurements, Biomedical Optics Express 4 (2013), 2629-2643.

[22] Z. Yuan and J. Ye, Fusion of fNIRS and fMRI: Identification when and where hemodynamic responses are changing in human brain, Frontier in Human Neurosciences 7 (2013), 676-684. 